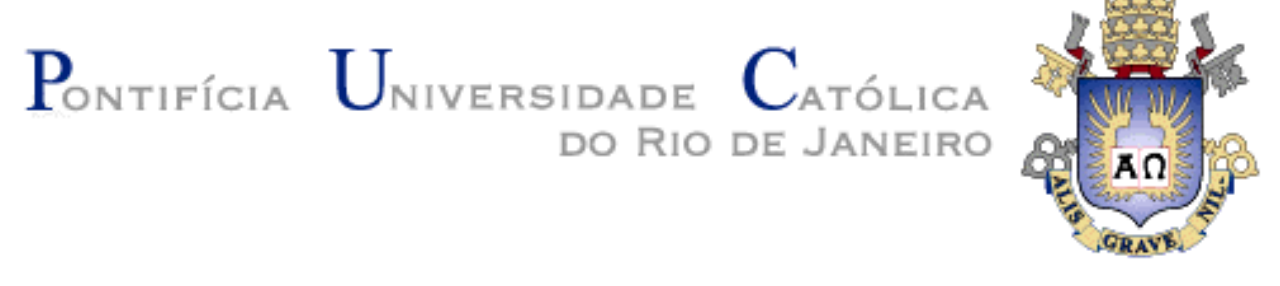

Cátia Maria Dias Ferreira

\title{
Avaliação da Metacomunicação Intercultural na Interação Humano-Computador: um vocabulário para acessar as perspectivas culturais dos usuários
}

\section{Dissertação de Mestrado}

Dissertação apresentada como requisito parcial para obtenção do grau de Mestre pelo Programa de Pós-Graduação em Informática do Departamento de Informática do Centro Técnico e Científico da PUC-Rio.

Orientadora: Prof ${ }^{a}$. Clarisse Sieckenius de Souza 


\section{Pontifícia U Uiversidade $C_{\text {atólica }}$}

Cátia Maria Dias Ferreira

\section{Avaliação da Metacomunicação Intercultural na Interação Humano-Computador: um vocabulário para acessar as perspectivas culturais dos usuários}

Dissertação apresentada como requisito parcial para obtenção do grau de Mestre pelo Programa de Pós-Graduação em Informática do Departamento de Informática do Centro Técnico e Científico da PUC-Rio. Aprovada pela Comissão Examinadora abaixo assinada.

Profa. . Clarisse Sieckenius de Souza

Orientadora

Departamento de Informática - PUC-Rio

Prof ${ }^{\mathrm{a}}$. Luciana Cardoso de Castro Salgado

Departamento de Informática - PUC-Rio

Prof ${ }^{\text {. Simone Diniz Junqueira Barbosa }}$

Departamento de Informática - PUC-Rio

Prof ${ }^{\mathrm{a}}$. Raquel Oliveira Prates

Departamento de Ciência da Computação - UFMG

Prof. José Eugenio Leal

Coordenador Setorial do Centro Técnico Científico - PUC-Rio

Rio de Janeiro, 31 de março de 2014 
Todos os direitos reservados. É proibida a reprodução total ou parcial do trabalho sem autorização da universidade, da autora e do orientador.

\section{Cátia Maria Dias Ferreira}

Bacharel em Estatística pela Universidade do Estado do Rio de Janeiro (UERJ) em 1989 e Pós-Graduação em Análise, Gerência e Projeto de Sistemas pela Pontifícia Universidade Católica do Rio de Janeiro (PUC-Rio) em 1996.

Ficha Catalográfica

Ferreira, Cátia Maria Dias

Avaliação da metacomunicação intercultural na interação humano-computador: um vocabulário para acessar as perspectivas culturais dos usuários / Cátia Maria Dias Ferreira; orientadora: Clarisse Sieckenius de Souza. - 2014.

$195 \mathrm{f}$ : il. (color.) ; $30 \mathrm{~cm}$

Dissertação (mestrado) - Pontifícia Universidade Católica do Rio de Janeiro, Departamento de Informática, 2014.

Inclui bibliografia

1. Informática - Teses. 2. Avaliação de IHC. 3 . Cultura. 4. Metáforas de perspectivas culturais. 5. Engenharia semiótica. 6. IHC e cultura. I. Souza, Clarisse Sieckenius de. II. Pontifícia Universidade Católica do Rio de Janeiro. Departamento de Informática. III. Título.

CDD: 004 
Aos meus pais, Oberdan e Inês, com amor e carinho. 


\section{Agradecimentos}

A Deus, por estar sempre comigo, me permitindo superar os desafios da vida.

Aos meus queridos pais Oberdan e Inês e aos meus irmãos por todo o carinho, amor e apoio durante todo o mestrado.

Ao meu namorado Rigan, pelo apoio e carinho que sempre teve comigo. Obrigada pela paciência em todos os momentos e pelo interesse no meu trabalho.

Aos familiares e todos os amigos que compreenderam a minha ausência e torceram por mim nessa jornada.

À amiga Patrícia Tavares, que sempre esteve presente, obrigada pela sua amizade e compreensão. À minha querida sobrinha Tainã, meu carinho e gratidão. À amiga Tereza Carnevale que me ajudou em um momento fundamental desse mestrado.

À professora Clarisse, minha orientadora, pela oportunidade que me foi dada. Sou imensamente grata por isso e pelo privilégio em compartilhar da sua sabedoria. Agradeço a sua confiança e entusiasmo com a minha pesquisa.

À professora Luciana, pela competência com que conduziu esse trabalho, pelas suas críticas sempre construtivas, respeito, dedicação, paciência e palavras de incentivo.

Às professoras Simone e Raquel, membros da banca examinadora, pelos valiosos conselhos e recomendações sobre este trabalho.

Aos amigos do SERG (Ingrid, Cleyton, Juliana, Eduardo, Marcelle, João, Luiz Marques, Hugo, Priscilla e Greis) e em especial, a Ingrid que sempre esteve pronta para me ajudar nos mais diversos assuntos.

Aos amigos da PUC-Rio, Ximena, Adriano, Lívia, Albaney e Marcelo que fizeram parte dessa conquista.

A todas as pessoas que gentilmente e voluntariamente participaram dos estudos que realizei nessa pesquisa e que por questões éticas não posso citar os nomes.

Finalmente ao IBGE pela concessão do afastamento e à PUC-Rio e CAPES pelo suporte financeiro recebido durante a pesquisa. 


\section{Resumo}

Ferreira, Cátia Maria Dias Ferreira; de Souza, Clarisse Sieckenius (Orientadora). Avaliação da Metacomunicação Intercultural na Interação Humano-Computador: um vocabulário para acessar as perspectivas culturais dos usuários. Rio de Janeiro, 2014. 195p. Dissertação de Mestrado - Departamento de Informática, Pontifícia Universidade Católica do Rio de Janeiro.

É fato que a diversidade cultural tornou-se um novo desafio na Interação Humano-Computador. Atualmente, os usuários podem navegar por quase todos os "lugares" da Web, sem fronteiras nacionais e/ou culturais, fazendo contato intencional ou não com elementos de culturas estrangeiras (idiomas, práticas e etc.). A Web tornou-se, portanto, um local privilegiado para encontros interculturais, i.e., um lugar onde os usuários têm a oportunidade de entrar em contato com a diversidade cultural diretamente (ao interagir com outros usuários através de redes sociais, por exemplo) ou indiretamente (quando interagem com aplicações que carregam traços culturais estrangeiros). Este cenário indica a necessidade de uma investigação sobre como a metacomunicação (comunicação sobre a comunicação) intercultural dos designers para os usuários é percebida pelos usuários. Com isto objetivamos entender como os usuários percebem as oportunidades de estabelecer contato com a diversidade cultural ao interagirem com aplicações multiculturais e como essas percepções podem contribuir em atividades de Avaliação de IHC de tais sistemas. Então, a fim de investigar se e como os usuários expressam as suas percepções e reações sobre as oportunidades promovidas pelos encontros interculturais indiretos, realizamos estudos empíricos onde oferecemos um vocabulário específico (Metáforas de Perspectivas Culturais). Realizamos também outros estudos sem oferecer nenhum vocabulário, i.e., deixamos os usuários falarem livremente sobre as oportunidades de fazer contato com a diversidade cultural na Interação Humano-Computador. Esses estudos foram realizados no contexto de duas aplicações multiculturais (uma do domínio linguístico e outra de um domínio não linguístico). Entre os resultados 
obtidos, destaca-se o potencial do vocabulário específico no ciclo de design de interação de sistemas multiculturais, revelando que as Metáforas de Perspectivas Culturais são uma ferramenta de apoio promissora para as práticas de design participativo, i.e., um meio de expressão e comunicação para os usuários qualificarem suas experiências de interação reais ou potenciais.

\section{Palavras-chave}

Avaliação de IHC; Cultura; Metáforas de Perspectivas Culturais; Engenharia Semiótica; IHC e Cultura. 


\section{Abstract}

Ferreira, Cátia Maria Dias Ferreira; de Souza, Clarisse Sieckenius (Advisor). Intercultural Metacommunication Evaluation in HumanComputer Interaction: A Vocabulary to Access Users' Cultural Perspectives. Rio de Janeiro, 2014. 195p. MSc. Dissertation Departamento de Informática, Pontifícia Universidade Católica do Rio de Janeiro.

It is fact that cultural diversity has become a new challenge in HumanComputer Interaction. Today users can navigate almost anywhere on the Web, with no cultural and/or national boundaries, having intentional or unintentional contact with foreign cultural elements (languages, practices etc.). Therefore, the Web became a privileged place for intercultural encounters, i.e., a place where users have the opportunity to be in contact with cultural diversity directly (when interacting with other users using social networks, for example) or indirectly (when interacting with applications, which have foreign cultural features). This scenario indicates the need for an investigation about how the intercultural metacommunication (communication about communication) of designers to users is noticed by users. Hereupon, we intend to understand how users perceive the opportunities to make contact with cultural diversity when interacting with crosscultural applications and how these perceptions can contribute in the HCI Evaluation activities of these systems. Thus, in order to investigate whether and how users express their perceptions and reactions over opportunities promoted by indirect intercultural encounters, we conducted empirical studies in which we offered a specific vocabulary (Cultural Viewpoint Metaphors). We also conducted other studies without offering any vocabulary, that is, we let users speak freely about the opportunities to make contact with cultural diversity in HumanComputer Interaction. These studies were conducted in the context of two crosscultural applications (one in a linguistic domain and one in a non-linguistic domain). Among the results obtained, it was highlighted the potential of the specific vocabulary in the interaction design cycle of cross-cultural systems, 
revealing that Cultural Viewpoint Metaphors are a promising supporting tool for participatory design practices, i.e., a medium of expression and communication for users to qualify their real or potential interaction experiences.

\section{Keywords}

HCI Evaluation; Culture; Cultural Viewpoint Metaphors; Semiotic Engineering; HCI and Culture. 


\section{Sumário}

1 Introdução 17

$\begin{array}{lll}1.1 & \text { Motivações e Objetivos } & 17\end{array}$

1.2 A organização da dissertação 22

2 Conceituação 23

2.1 Engenharia Semiótica e Cultura 23

2.2 Metáforas de Perspectivas Culturais (CVM) 30

$3 \quad$ Trabalhos Relacionados $\quad 37$

3.1 O processo de design de IHC e Cultura 37

3.1.1. Estudos que investigam a adaptação cultural das interfaces $\begin{array}{ll}\text { no processo de design } & 37\end{array}$

3.1.2. Estudos sobre os métodos e práticas de IHC 39

3.1.2.1. Design Participativo no contexto de IHC e cultura 39

3.1.2.2. Usabilidade no contexto de IHC e cultura 40

3.1.2.3. Comunicabilidade no contexto de IHC e cultura 42

3.1.3. Estudos que apresentam uma revisão da literatura sobre o design de IHC e cultura 44

$4 \quad$ Estudos empíricos 46

4.1 Metodologia 46

4.1.1. Questões de Pesquisa e Objetivos 46

4.1.2. Websites usados nos estudos 51

4.1.3. Estudos com CVM 52

4.1.4. Estudos sem CVM 54

4.1.5. Quadro comparativo das etapas de pesquisa dos estudos 57

4.2 Estudo 1: Domínio Linguístico com CVM 58 
4.2.1. Resultados detalhados do E1

62

4.2.1.1. Análise das situações de aprendizado, utilizando o vocabulário CVM

62

4.2.1.2. Análise do contato intercultural através da língua 65

4.2.1.3. Evidências das oportunidades vislumbradas em tempo de avaliação

67

4.2.1.4. Dificuldades encontradas durante a atividade de avaliação $\quad 70$

4.2.2. Síntese dos resultados do E1 70

4.3 Estudo 2: Domínio Linguístico sem CVM 72

4.3.1. Resultados detalhados do E2 74

4.3.1.1. Percepção da outra cultura durante a interação 74

4.3.1.2. Foco no conteúdo 78

4.3.1.3. Contato com as próprias heranças culturais ao avaliar o encontro intercultural $\quad 80$

4.3.1.4. Dificuldades encontradas durante a atividade de avaliação $\quad 80$

4.3.1.5. Reflexões sobre a atividade de avaliação 81

4.3.1.6. Uso do vocabulário próprio em tempo de avaliação 84

4.3.2. Síntese dos resultados do E2 89

4.4 Estudo 3: Domínio não Linguístico com CVM 91

4.4.1. Resultados detalhados do E3 94

4.4.1.1. Análise das situações de uso, utilizando o vocabulário CVM 94

4.4.1.2. Evidências que as CVM ajudam a revelar as estratégias comunicativas em tempo de avaliação $\quad 97$

4.4.1.3. Evidências que podem influenciar na percepção das CVM em tempo de avaliação $\quad 100$

4.4.1.4. Análise do contato intercultural através da língua 103

4.4.1.5. Dificuldades encontradas durante a atividade de avaliação 107

4.4.1.6. Análise das descobertas usando as CVM em tempo de avaliação 108

4.4.2. Síntese dos resultados do E3 115

4.5 Estudo 4: Domínio não Linguístico sem CVM 117

4.5.1. Resultados detalhados do E4 119 
4.5.1.1. Percepção da outra cultura durante a interação

$\begin{array}{ll}\text { 4.5.1.2. Foco no conteúdo } & 124\end{array}$

4.5.1.3. Dificuldades encontradas durante a atividade de avaliação 125

4.5.1.4. Reflexões sobre a atividade de avaliação 127

4.5.1.5. Uso do vocabulário próprio em tempo de avaliação 131

4.5.2. Síntese dos resultados do E4 138

$\begin{array}{lll}4.6 & 139\end{array}$

4.6.1. Resultados da Triangulação dos Estudos 1 e $2 \quad 140$

4.6.1.1. Análise das situações de aprendizado, utilizando o $\begin{array}{ll}\text { vocabulário CVM } & 141\end{array}$

4.6.1.2. Análise do contato intercultural através da língua 144

4.6.1.3. Evidências das oportunidades vislumbradas em tempo

$\begin{array}{ll}\text { de avaliação } & 146\end{array}$

4.6.1.4. Dificuldades encontradas durante a atividade de avaliação 148

4.6.2. Contrastando os resultados de E1 e E2 148

4.6.3. Resultados da Triangulação dos Estudos 1 e 3

4.6.3.1. Análise das situações de aprendizado, utilizando o vocabulário CVM 154

4.6.3.2. Análise do contato intercultural através da língua 156

4.6.3.3. Evidências das oportunidades vislumbradas em tempo $\begin{array}{ll}\text { de avaliação } & 159\end{array}$

4.6.3.4. Dificuldades encontradas durante a atividade de avaliação 162

4.6.4. Contrastando os resultados de E1 e E3 162

$\begin{array}{ll}\text { 4.6.5. Discussão } & 166\end{array}$

5 Conclusão 167

$\begin{array}{lll}5.1 & \text { Contribuições } & 169\end{array}$

5.1.1. IHC no design de sistemas multiculturais 169

5.1.2. Contribuições para a Engenharia Semiótica e Cultura 171

$\begin{array}{lll}5.2 & \text { Trabalhos Futuros } & 171\end{array}$

$6 \quad$ Referências Bibliográficas $\quad 174$ 
Apêndice A Termo de Consentimento do Estudo 1

Apêndice B Termo de Consentimento do Estudo 2

Apêndice C Termo de Consentimento do Estudo 3

Apêndice D Termo de Consentimento do Estudo 4

Apêndice E Resumo das CVM

Apêndice F Perguntas da Entrevista Pós-Teste

Apêndice G Cenários de Uso dos Estudos 1 e 2

Apêndice H Cenários de Uso dos Estudos 3 e 4 


\section{Lista de Figuras}

Figura 1: Espaço de Design da Engenharia Semiótica

Figura 2: Espaço de Design da Engenharia Semiótica com a Inclusão da diversidade cultural $\quad 27$

Figura 3: Encontro Intercultural direto (através do Facebook) 28

Figura 4: Encontro Intercultural indireto (Avis) 28

Figura 5: Encontro Intercultural indireto (Englishtown@) 29

Figura 6: Continuum de Aproximação Cultural 31

Figura 7: Continuum de Aproximação Cultural (Ausência da Mediação Cultural) 31

Figura 8: Continuum de Aproximação Cultural (Mediação Cultural) 32

Figura 9: Efeitos das metáforas na organização do discurso interativo 33

Figura 10: Metáfora Viajante Doméstico (Localização) - Rio 2016

Figura 11: Metáfora Estrangeiro com Tradutor (Localização) Wikipedia (em português) 35

Figura 12: Visão geral da pesquisa $\quad 51$

Figura 13: Englishtown@ website $\quad 51$

Figura 14: Wikipedia website $\quad 52$

Figura 15: Etapas dos Estudos com CVM 53

Figura 16: Etapas dos Estudos sem CVM 54

Figura 17: Vídeo para aprender Direções em inglês e Artigo

para aprender Protocolo Social $\quad 59$

Figura 18: Lição para aprender Inglês para Negócios e Quiz sobre Lugares de língua inglesa $\quad 59$

Figura 19: Apresentação do primeiro estudo aos participantes 60

Figura 20: Estudo 1 (Cenário 2) 61

Figura 21: Estudo 1 (Cenário 6) 61

Figura 22: Resultado do quiz (Situação de Negócios) 63

Figura 23: Trecho do vídeo (Aprenda inglês em Sidney) 64

Figura 24: Trecho do artigo (Como perguntar onde fica o

banheiro em Inglês) $\quad 64$ 
Figura 25: Trecho do artigo (Selecionando um candidato para uma entrevista) 65

Figura 26: Trecho do artigo (Como dizer Não em Inglês) 66

Figura 27: Trecho do quiz (Seu Inglês é bom o suficiente para se virar em Londres?) 66

Figura 28: Apresentação do segundo estudo aos participantes 73

Figura 29: Exemplo usado na Mini Avaliação do Estudo 2

(Englishtown@)

Figura 30: Trecho (Cursos no Exterior) 76

Figura 31: Culinária da Mazóvia 92

Figura 32: Cenário 4 (Wikipedia) 93

Figura 33: Cenário 1 (Wikipedia) 93

Figura 34: Trecho do Cenário 7 (Wikipedia) 96

Figura 35: Trecho da Culinária da Europa (Cenário 5) 97

Figura 36: Trecho com a dica "Culinária da Alemanha" (Cenário 5) 97

Figura 37: Trecho do artigo sobre o Toque de angola no berimbau (Cenário 8) 98

Figura 38: Trecho da página da Wikipedia em polonês (Cenário 2) 98

Figura 39: Trecho do Cenário 3 (Wikipedia) 98

Figura 40: Linha de scrimmage e campo do Futebol Americano em jardas (Wikipedia) 99

Figura 41: Trecho da página do Futebol Americano em Português (Cenário 9) 105

Figura 42: Trecho da página em polonês da Wikipedia (Cenário 2) 106

Figura 43: Trecho da página principal da Wikipedia (Cenário 1) 107

Figura 44: Trecho "Fazer avançar a bola" (Wikipedia) 111

Figura 45: Trecho da página da Wikipedia em chinês 116

Figura 46: Exemplo usado na Mini Avaliação do Estudo 2

(Wikipedia) 118

Figura 47: Trecho sobre a culinária japonesa (Wikipedia) 121

Figura 48: Trecho do artigo sobre futebol americano (Índice) 123

Figura 49: Trecho da página principal da Wikipedia (pesquisa) 123

Figura 50: Trecho da página principal da Wikipedia em português 124 


\section{Lista de Tabelas}

Tabela 1: Framework das CVM 33

Tabela 2: Estudos Empíricos $\quad 47$

Tabela 3: Resumo da Etapa 2 dos Estudos Empíricos 58

Tabela 4: Resumo da Triangulação Endógena (E1 e E2) 152

Tabela 5: Resumo da Triangulação Exógena (E1 e E3) 165 